\title{
Double resonance rotational spectroscopy of $\mathrm{CH}_{2} \mathrm{D}^{+}$
}

\author{
Matthias Töpfer, Pavol Jusko, Stephan Schlemmer, and Oskar Asvany \\ I. Physikalisches Institut, Universität zu Köln, Zülpicher Str. 77, 50937 Köln, Germany
e-mail: asvany@ph1.uni-koeln.de \\ Received 1 June 2016 / Accepted 16 August 2016

\begin{abstract}
Context. Deuterated forms of $\mathrm{CH}_{3}^{+}$are thought to be responsible for deuterium enrichment in lukewarm astronomical environments. There is no unambiguous detection of $\mathrm{CH}_{2} \mathrm{D}^{+}$in space to date.

Aims. Four submillimetre rotational lines of $\mathrm{CH}_{2} \mathrm{D}^{+}$are documented in the literature. Our aim is to present a complete dataset of highly resolved rotational lines, including millimetre $(\mathrm{mm})$ lines needed for a potential detection.

Methods. We used a low-temperature ion trap and applied a novel IR-mm-wave double resonance method to measure the rotational lines of $\mathrm{CH}_{2} \mathrm{D}^{+}$.

Results. We measured 21 low-lying $(J \leq 4)$ rotational transitions of $\mathrm{CH}_{2} \mathrm{D}^{+}$between $23 \mathrm{GHz}$ and $1.1 \mathrm{THz}$ with accuracies close to $2 \mathrm{ppb}$.
\end{abstract}

Key words. astrochemistry - line: identification - molecular data - ISM: molecules - radio lines: ISM

\section{Introduction}

According to current astrochemical models (Roueff et al. 2007; Parise et al. 2009), $\mathrm{CH}_{3}^{+}$and its deuterated analogues are thought to be responsible for deuteration processes at lukewarm temperatures $(\sim 20 \mathrm{~K}<T<\sim 80 \mathrm{~K})$ following the reaction chain (Smith et al. 1982; Asvany et al. 2004)

$$
\begin{aligned}
& \mathrm{CH}_{3}^{+}+\mathrm{HD} \rightleftharpoons \mathrm{CH}_{2} \mathrm{D}^{+}+\mathrm{H}_{2} \\
& \mathrm{CH}_{2} \mathrm{D}^{+}+\mathrm{HD} \rightleftharpoons \mathrm{CD}_{2} \mathrm{H}^{+}+\mathrm{H}_{2} \\
& \mathrm{CD}_{2} \mathrm{H}^{+}+\mathrm{HD} \rightleftharpoons \mathrm{CD}_{3}^{+}+\mathrm{H}_{2} .
\end{aligned}
$$

Unfortunately, this hypothesis rests on weak grounds, as none of the involved cationic species is detected in space, and there are only searches and tentative detections of $\mathrm{CH}_{2} \mathrm{D}^{+}$ by Wootten $\&$ Turner (2008) and Roueff et al. (2013). Those searches and tentative detections used predicted $\mathrm{CH}_{2} \mathrm{D}^{+}$transition frequencies based on laboratory data available at that time. The first predictions of the rotational transition frequencies were provided by the IR data by Rösslein et al. (1991) and Jagod et al. (1992), and those were later refined by the high-resolution IR work by Gärtner et al. (2010, 2013) and, in particular, by the first four pure rotational lines measured by Amano (2010). More recently, two fundamental rotational transitions were measured in the Cologne laboratories (Brünken et al. 2014b) using a helium attachment technique at $4 \mathrm{~K}$ (Brünken et al. 2014a). In the work presented here, a double resonance spectroscopy technique is used to measure 21 rotational transitions of $\mathrm{CH}_{2} \mathrm{D}^{+}$between $23 \mathrm{GHz}$ and $1.1 \mathrm{THz}$ with close to $2 \mathrm{ppb}$ accuracy. With this complete dataset, we hope to trigger a renewed search for $\mathrm{CH}_{2} \mathrm{D}^{+}$.

\section{Laboratory methods}

The experiment was performed in a $4 \mathrm{~K}$ 22-pole ion trapping machine, which is described in Asvany et al. (2014); the applied IRmillimetre-wave (mm-wave) double resonance technique was first mentioned in Gärtner et al. (2013) and later documented by Jusko et al. (2014). In brief, $\mathrm{CH}_{2} \mathrm{D}^{+}$ions were produced by ionizing $\mathrm{CH}_{3} \mathrm{D}$ gas in a storage ion source. The ions were mass selected and a few 10000 ions were injected into the 22-pole ion trap (Asvany et al. 2010). On entrance, the ion ensemble was cooled down to the ambient trap temperature by a short helium gas pulse. The trap temperature was adjusted between 9 and $25 \mathrm{~K}$, depending on the probed rotational level. During the trapping time of about $700 \mathrm{~ms}$, the ion ensemble was exposed to cold hydrogen gas. The backwards direction of reaction (1) was induced by vibrationally exciting the stored ions, leading to a detectable $\mathrm{CH}_{3}^{+}$signal. This process is called laser induced reaction (LIR; Schlemmer et al. 1999, 2002). For this vibrational excitation, a cw optical parametric oscillator (OPO) was held in resonance (within $\pm 2 \times 10^{-4} \mathrm{~cm}^{-1}$ ) on selected transitions of the $v_{1}$ or $v_{4}$ bands (Jagod et al. 1992; Gärtner et al. 2013). With the IR frequency held on resonance, a cw (sub)mm-wave beam was shone simultaneously onto the trapped ion ensemble. A mirror with a hole was used to superpose the two beams, as shown in Fig. 1 of Jusko et al. (2014). The two coaxial beams then entered the vacuum chamber via a diamond window and reached the $6.6 \mathrm{~mm}$ diameter aperture of the 22-pole ion trap. For the generation of long wavelength radiation, synthesizers (Agilent E8257D or Rohde \& Schwarz SMF 100A) with suitable horn antennas were used, whereas a modular chain consisting of amplifiers and multipliers (WR9.0M-AMC, Virginia Diodes Inc.) was applied for frequencies beyond $80 \mathrm{GHz}$. As shown in the inset of Fig. 1, the rotational excitation pumps $\mathrm{CH}_{2} \mathrm{D}^{+}$ions into the state probed by the vibrational transition, increasing the $\mathrm{CH}_{3}^{+}$signal count. By scanning the rotational excitation frequency over the resonance (symmetrically up and down), a rotational line can be obtained.

\section{Experimental results}

Owing to the existing predictions, no tedious searches were necessary and the targeted lines were usually found within minutes. 


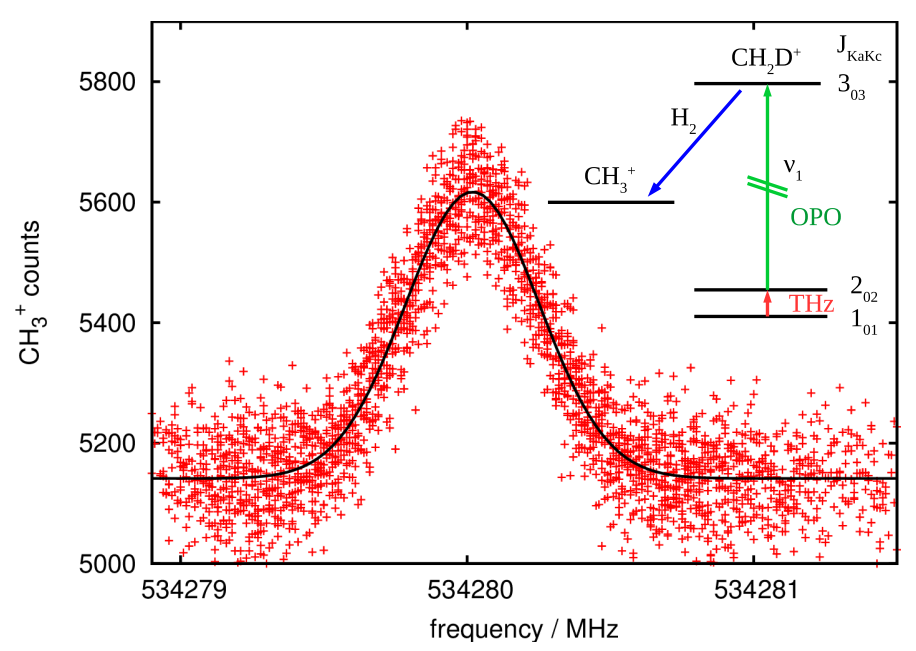

Fig. 1. Measurement of the $2_{02} \leftarrow 1_{01}$ rotational ground state transition with the IR-mm-wave double resonance technique at $11 \mathrm{~K}$. The energy level diagram (inset) explains the double resonance method: with the IR light held in resonance (OPO; long green arrow) at $3029.6352 \mathrm{~cm}^{-1}$, more than $5100 \mathrm{CH}_{3}^{+}$signal counts are obtained. When the submmwave source (THz, small red arrow) is tuned into resonance, additional $\mathrm{CH}_{2} \mathrm{D}^{+}$ions are pumped into the 202 rotational level, leading to an increase of the signal by about 500 counts. In this example, the submmwave source has been tuned up and down six times, stepping $10 \mathrm{kHz}$ after each $1 \mathrm{~s}$ trapping cycle. A Gaussian fit of the seen signal gives a centre frequency of $534280.0217(24) \mathrm{MHz}$ and a kinetic temperature of about $30 \mathrm{~K}$. Eight such measurements lead to the final value given in Table 1.

A sample measurement of the transition $2_{02} \leftarrow 1_{01}$ is shown in Fig. 1 together with a Gaussian fit and more explanations. To improve the statistics, such measurements were typically repeated five to ten times. In total we detected 21 rotational lines. These are summarized in Table 1 and are compared to the 4 rotational lines measured by Amano (2010), whose conservative error limits are confirmed and improved by at least one order of magnitude in this work. Surprisingly, our measured values also agree extremely well with the very first prediction of rotational transitions of $\mathrm{CH}_{2} \mathrm{D}^{+}$given by Rösslein et al. (1991).

The cold temperatures and good signal-to-noise ratio in this work usually permit us to determine the transitions with uncertainties close to $2 \mathrm{ppb}$. Transitions with higher uncertainties in the table stem either from weakly populated levels (e.g. $4_{13} \leftarrow 4_{14}$ ), or are transitions with weak intrinsic transition strength $\left(\Delta K_{a}=2\right.$, e.g. $3_{22} \leftarrow 3_{02}$ or $\left.2_{21} \leftarrow 2_{02}\right)$ and/or with limited submm-wave source power (in upper frequency range, e.g. $4_{23} \leftarrow 3_{22}$ ). We also want to point out the low frequency transitions, for which the unresolved hyperfine splittings, caused mainly by the electrical quadrupole moment of the deuterium nucleus, start broadening and skewing the Doppler line shapes; see Figs. 2 and 3 for the $23 \mathrm{GHz}$ and $67 \mathrm{GHz}$ lines, respectively. For these lines, some residual power broadening effects cannot be excluded. As simple simulations of the hyperfine structure did not fully recover the seen structures, we kept using Gaussian functions, leading to less reliable definitions of the line centres given in Table 1. But because of the large dataset given in Table 1, the line centres and their error limits can be checked by forming combination differences. For instance, the difference between the rotational levels $2_{11}$ and $3_{13}$ can be formed in two different ways (see level scheme in Fig. 4 and Table A.1), and shows that our data is reasonable. A full list of 32 such combination differences is given in Table A.1. In this

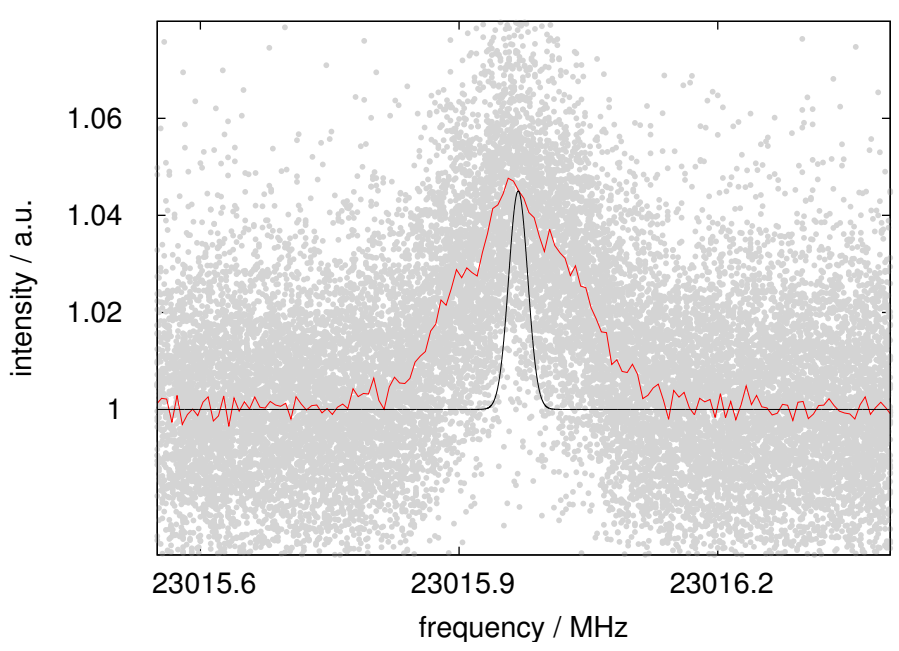

Fig. 2. Transition $2_{20} \leftarrow 2_{21}$ at $23 \mathrm{GHz}$ measured at a nominal trap temperature of $15 \mathrm{~K}$. The broadness of this feature can be recognized by comparison with the narrow Gaussian curve, which is the shape expected for a single line at $40 \mathrm{~K}$. Since pressure or Doppler broadening to this extent can be excluded, unresolved hyperfine structure is the most reasonable explanation for this broadening, whereas some residual power broadening effect cannot be excluded. These hyperfine splittings are dominated by the small electrical quadrupole moment of the deuterium nucleus. To better identify the structure in this feature, the data of 13 single measurements (shown as pale grey dots) have been normalized (to the off-resonant $\mathrm{CH}_{3}^{+}$counts), averaged, and binned into $6 \mathrm{kHz}$ steps. The result is shown as a red curve, where two smaller peaks become discernible on both sides of the feature.

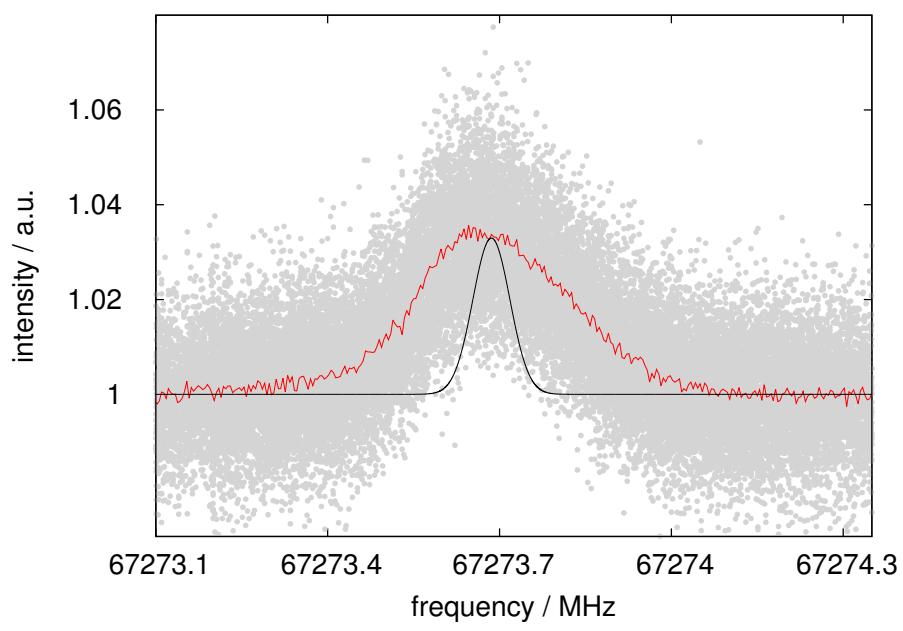

Fig. 3. Transition $1_{10} \leftarrow 1_{11}$ at $67 \mathrm{GHz}$ measured at a nominal temperature of $9 \mathrm{~K}$. Similar to Fig. 2, the narrow Gaussian is the shape expected for a single line at $40 \mathrm{~K}$. Averaging over several measurements and binning into $4 \mathrm{kHz}$ steps yields the red curve, revealing deviations from a Gaussian shape.

list, the combination differences involving the $67 \mathrm{GHz}$ line show deviations beyond the expected level, the most probable reason being the unresolved hyperfine structure mentioned above. Thus, excluding this line, our data were used as input to an asymmetric rotor model (Western 2016). Transitions from previous publications (Jagod et al. 1992; Amano 2010; Gärtner et al. 2013) were not included here as they did not significantly improve the ground state constants. The obtained rotational constants for the ground state of $\mathrm{CH}_{2} \mathrm{D}^{+}$are summarized in Table 2 . 
Table 1. Measurements of low-lying $(J \leq 4)$ rotational transitions (in $\mathrm{MHz}$ ) of $\mathrm{CH}_{2} \mathrm{D}^{+}$up to $1.1 \mathrm{THz}$ (for comparison, the hitherto available discharge cell measurements of Amano 2010 are also listed).

\begin{tabular}{lrcl}
\hline \hline$J_{K_{a}^{\prime} K_{c}^{\prime}}^{\prime} \leftarrow J_{K_{a} K_{c}}$ & This work & Amano (2010) \\
\hline $2_{20}$ & $2_{21}$ & $23015.9690(12)$ & \\
$1_{10}$ & $1_{11}$ & $67273.6872(11)$ & \\
$3_{21}$ & $3_{22}$ & $101483.1327(5)$ & \\
$2_{11}$ & $2_{12}$ & $201754.0373(5)$ & \\
$4_{22}$ & $4_{23}$ & $251007.7368(42)$ & \\
$1_{01}$ & $0_{00}$ & $278691.7708(9)$ & \\
$3_{12}$ & $3_{13}$ & $398038.7155(11)$ & \\
$2_{12}$ & $1_{11}$ & $490012.2588(7)$ & $490012.247(30)$ \\
$2_{02}$ & $1_{01}$ & $534280.0198(11)$ & \\
$2_{21}$ & $2_{02}$ & $588663.4054(14)$ & \\
$2_{11}$ & $1_{10}$ & $624492.6351(9)$ & $624492.648(20)$ \\
$4_{13}$ & $4_{14}$ & $637667.8351(57)$ & \\
$3_{22}$ & $3_{03}$ & $666869.7354(38)$ & \\
$3_{13}$ & $2_{12}$ & $722354.6106(9)$ & $722354.622(25)$ \\
$3_{03}$ & $2_{02}$ & $757258.1056(11)$ & \\
$3_{22}$ & $2_{21}$ & $835464.4209(47)$ & $835464.376(50)$ \\
$3_{21}$ & $2_{20}$ & $913931.5916(19)$ & \\
$3_{12}$ & $2_{11}$ & $918639.2934(20)$ & \\
$4_{14}$ & $3_{13}$ & $945398.0638(15)$ & \\
$4_{04}$ & $3_{03}$ & $963380.5889(18)$ & \\
$4_{23}$ & $3_{22}$ & $1095332.1756(153)$ & \\
\hline & & & \\
\hline
\end{tabular}

Notes. Uncertainties are given in parentheses.

Table 2. Best-fit spectroscopic parameters for the ground state of $\mathrm{CH}_{2} \mathrm{D}^{+}$(in $\mathrm{MHz}$ ) obtained using 20 rotational lines of this work.

\begin{tabular}{lrlr}
\hline \hline Symbol & Value & Symbol & Value \\
\hline$A$ & $280862.61(4)$ & $\Phi_{J}$ & $0.000219(4)$ \\
$B$ & $173019.812(3)$ & $\Phi_{J K}$ & \\
$C$ & $105686.555(3)$ & $\Phi_{K J}$ & $0.0058(1)$ \\
$\Delta_{J}$ & $3.64968(8)$ & $\Phi_{K}$ & \\
$\Delta_{J K}$ & $10.8915(5)$ & $\phi_{J}$ & $0.000041(3)$ \\
$\Delta_{K}$ & $8.089(9)$ & $\phi_{J K}$ & $0.00358(3)$ \\
$\delta_{J}$ & $1.39841(5)$ & $\phi_{K}$ & $-0.0069(3)$ \\
$\delta_{K}$ & $12.103(2)$ & & \\
\hline
\end{tabular}

Notes. Only parameters of statistical significance are listed.

\section{Conclusions and outlook}

While the IR-mm-wave double resonance technique has been applied before on single fundamental transitions of $\mathrm{H}_{2} \mathrm{D}^{+}$ (Gärtner et al. 2013) and $\mathrm{OH}^{-}$(Jusko et al. 2014), its full power is unfolded by measuring the complete set of all low-lying rotational transitions of $\mathrm{CH}_{2} \mathrm{D}^{+}$up to $1.1 \mathrm{THz}$, thereby leading to highly accurate spectroscopic parameters. A level diagram summarizing the rotational transitions studied in this work is shown in Fig. 4. We hope to trigger renewed searches of $\mathrm{CH}_{2} \mathrm{D}^{+}$ in space given the complete set of rotational transitions. As the hyperfine structure might become important for the lower frequency transitions, we also want to encourage high-level

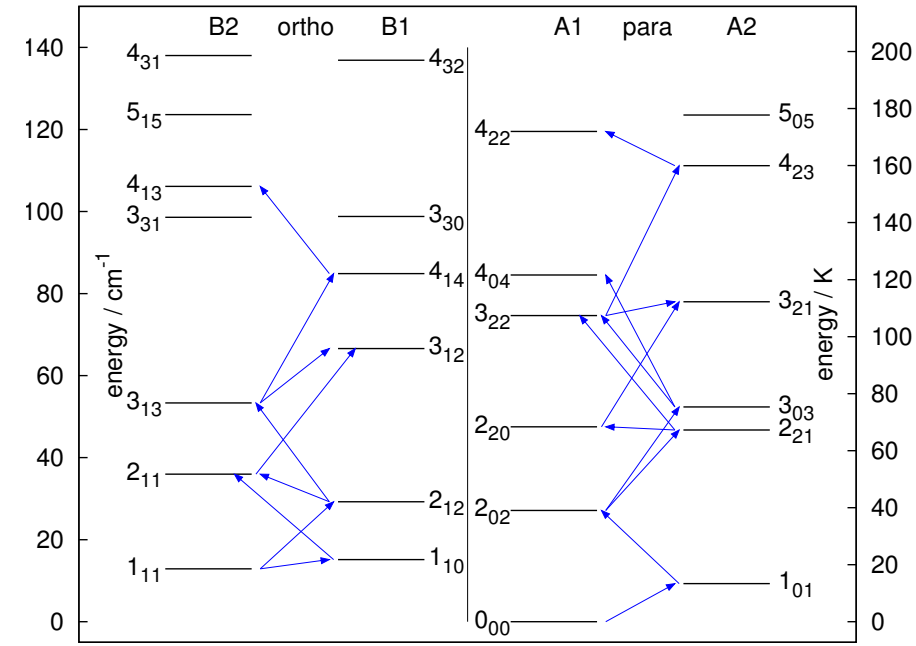

Fig. 4. Ground state rotational levels of $\mathrm{CH}_{2} \mathrm{D}^{+}$illustrating the 21 rotational transitions measured in this work.

ab initio calculations of the hyperfine structure, as carried out previously for $\mathrm{H}_{2} \mathrm{D}^{+}$and $\mathrm{D}_{2} \mathrm{H}^{+}$by Jensen et al. (1991, 1997). A full account of similar rotational experiments for $\mathrm{CD}_{2} \mathrm{H}^{+}$will be given in a later publication.

Acknowledgements. This work has been financially supported by the Deutsche Forschungsgemeinschaft (DFG) via SFB 956 project B2. P.J. is supported by the Alexander von Humboldt Stiftung. We thank Marius Hermanns and Sven Fanghänel for assistance in the experiment, as well as Sandra Brünken, Holger Müller, Sven Thorwirth, and Jürgen Gauß for valuable discussions.

\section{References}

Amano, T. 2010, A\&A, 516, L4

Asvany, O., Schlemmer, S., \& Gerlich, D. 2004, ApJ, 617, 658

Asvany, O., Bielau, F., Moratschke, D., Krause, J., \& Schlemmer, S. 2010, Rev. Sci. Instr., 81, 076102

Asvany, O., Brünken, S., Kluge, L., \& Schlemmer, S. 2014, Appl. Phys. B, 114, 203

Brünken, S., Kluge, L., Stoffels, A., Asvany, O., \& Schlemmer, S. 2014a, ApJ, 783, L4

Brünken, S., Kluge, L., Stoffels, A., \& Schlemmer, S. 2014b, unpublished results Gärtner, S., Krieg, J., Klemann, A., Asvany, O., \& Schlemmer, S. 2010, A\&A, 516, L3

Gärtner, S., Krieg, J., Klemann, A., et al. 2013, J. Phys. Chem. A, 117, 9975

Jagod, M.-F., Rösslein, M., Gabrys, C. M., et al. 1992, J. Chem. Phys., 97, 7111

Jensen, P., Paidarová, I., Vojtík, J., \& Špirko, V. 1991, J. Mol. Spectr., 150, 137

Jensen, P., Paidarová, I., Špirko, V., \& Sauer, S. P. A. 1997, Mol. Phys., 91, 319

Jusko, P., Asvany, O., Wallerstein, A.-C., Brünken, S., \& Schlemmer, S. 2014, Phys. Rev. Lett., 112, 253005

Parise, B., Leurini, S., Schilke, P., et al. 2009, A\&A, 508, 737

Rösslein, M., Jagod, M. F., Gabrys, C. M., \& Oka, T. 1991, ApJ, 382, L51

Roueff, E., Parise, B., \& Herbst, E. 2007, AA\&A, 464, 245

Roueff, E., Gerin, M., Lis, D. C., et al. 2013, J. Phys. Chem. A, 117, 9959

Schlemmer, S., Kuhn, T., Lescop, E., \& Gerlich, D. 1999, Int. J. Mass Spectrom., 185,589

Schlemmer, S., Lescop, E. V., Richthofen, J., \& Gerlich, D. 2002, J. Chem. Phys., 117, 2068

Smith, D., Adams, N., \& Alge, E. 1982, J. Chem. Phys., 77, 1261

Western, C. M. 2016, PGOPHER, a Program for Simulating Rotational Structure, C. M. Western, University of Bristol, http://pgopher.chm. bris.ac.uk

Wootten, A., \& Turner, B. E. 2008, in Organic Matter in Space, eds. S. Kwok, \& S. Sanford, IAU Symp., 251, 334 


\section{Appendix A: combination differences}

From the 21 rotational lines measured for $\mathrm{CH}_{2} \mathrm{D}^{+}$, the 32 combination differences listed in Table A. 1 could be obtained by forming sums and differences (see also Fig. 4). The eight combination differences which could be formed in two different ways show that our rotational line centres and their errors are reasonable, except for the $67 \mathrm{GHz}$ line, which exhibits a deviation on the order of $20 \mathrm{kHz}$. This line is most probably affected by unresolved hyperfine structure (and power broadening effects).

Table A.1. The combination differences (CD) obtained by forming sums or differences of the two rotational transitions $\mathrm{T} 1$ and $\mathrm{T} 2$ are listed here in ascending order.

\begin{tabular}{|c|c|c|c|c|}
\hline $\mathrm{Up}$ & Low & $\mathrm{CD}$ & $\mathrm{T} 1$ & $\mathrm{~T} 2$ \\
\hline $3_{03}$ & 221 & $168594.6855(60)$ & 666870 & 835464 \\
\hline $3_{03}$ & $2_{21}$ & $168594.7002(18)$ & 588663 & 757258 \\
\hline $4_{04}$ & $3_{22}$ & $296510.8535(42)$ & 666870 & 963381 \\
\hline 212 & $1_{10}$ & $422738.5716(13)$ & 67274 & 490012 \\
\hline 212 & $1_{10}$ & $422738.5978(10)$ & 201754 & 624493 \\
\hline $3_{13}$ & $22_{11}$ & $520600.5733(10)$ & 201754 & 722355 \\
\hline $3_{13}$ & $2_{11}$ & $520600.5779(23)$ & 398039 & 918639 \\
\hline $4_{14}$ & $3_{12}$ & $547359.3483(19)$ & 398039 & 945398 \\
\hline 220 & 202 & $611679.3744(18)$ & 23016 & 588663 \\
\hline 2 & $1_{11}$ & $691766.2961(9)$ & 201754 & 490012 \\
\hline 211 & $1_{11}$ & $691766.3223(14)$ & 67274 & 624493 \\
\hline $3_{21}$ & $3_{03}$ & $768352.8681(38)$ & 101483 & 666870 \\
\hline $3_{22}$ & $2_{20}$ & $812448.4519(49)$ & 23016 & 835464 \\
\hline $3_{22}$ & $2_{20}$ & $812448.4589(20)$ & 101483 & 913932 \\
\hline 202 & $0_{00}$ & $812971.7906(14)$ & 278692 & 534280 \\
\hline $3_{21}$ & 221 & $936947.5536(47)$ & 101483 & 835464 \\
\hline $3_{21}$ & 221 & $936947.5606(22)$ & 23016 & 913932 \\
\hline $4_{23}$ & $3_{21}$ & $993849.0429(153)$ & 101483 & 1095332 \\
\hline $3_{12}$ & 212 & $1120393.3261(14)$ & 398039 & 722355 \\
\hline 312 & $2{ }_{12}$ & $1120393.3307(21)$ & 201754 & 918639 \\
\hline 221 & $1_{01}$ & $1122943.4252(18)$ & 534280 & 588663 \\
\hline $3_{13}$ & $1_{11}$ & $1212366.8694(11)$ & 490012 & 722355 \\
\hline $3_{03}$ & $1_{01}$ & $1291538.1254(16)$ & 534280 & 757258 \\
\hline $4_{22}$ & $3_{22}$ & $1346339.9124(159)$ & 251008 & 1095332 \\
\hline $3_{22}$ & 202 & $1424127.8263(49)$ & 588663 & 835464 \\
\hline $3_{22}$ & $2_{02}$ & $1424127.8410(40)$ & 666870 & 757258 \\
\hline $3_{12}$ & $1_{10}$ & $1543131.9285(22)$ & 624493 & 918639 \\
\hline $4_{13}$ & $3_{13}$ & $1583065.8989(59)$ & 637668 & 945398 \\
\hline $4_{14}$ & $2{ }_{12}$ & $1667752.6744(17)$ & 722355 & 945398 \\
\hline $4_{04}$ & 202 & $1720638.6945(21)$ & 757258 & 963381 \\
\hline $4_{23}$ & $3_{03}$ & $1762201.9110(158)$ & 666870 & 1095332 \\
\hline $4_{23}$ & $2_{21}$ & $1930796.5965(160)$ & 835464 & 1095332 \\
\hline
\end{tabular}

Notes. All values in MHz; uncertainties are given in parentheses. 